\title{
Acoustic attenuation probe for fermion superfluidity in ultracold atom gases
}

\author{
Sergio Gaudio, ${ }^{1,2}$, 田 Bogdan Mihaila, ${ }^{2}$ Krastan B. Blagoev, ${ }^{2}$ Kevin S. Bedell, ${ }^{1}$ and Eddy Timmermans ${ }^{2}$, 团 \\ ${ }^{1}$ Department of Physics, Boston College, Chestnut Hill, MA 02167 \\ ${ }^{2}$ Theoretical Division, Los Alamos National Laboratory, Los Alamos, NM 87545
}

\begin{abstract}
Dilute gas Bose-Einstein condensates (BEC's), currently used to cool fermionic atoms in atom traps, can also probe the superfluidity of these fermions. The damping rate of BEC-acoustic excitations (phonon modes), measured in the middle of the trap as a function of the phonon momentum, yields an unambiguous signature of BCS-like superfluidity, provides a measurement of the superfluid gap parameter and gives an estimate of the size of the Cooper-pairs in the BEC-BCS crossover regime. We also predict kinks in the momentum dependence of the damping rate which can reveal detailed information about the fermion quasi-particle dispersion relation.

PACS numbers: 03.75.Fi, 05.30.Jp, 32.80.Pj, 67.90.+z
\end{abstract}

Recently, cold atom physics entered [1] the previously inaccessible [2] Bose-Einstein condensate (BEC) regime of fermion superfluidity. A magnetic field sweep through a Feshbach resonance induced the fermions to pair up into diatomic molecules which subsequently occupied a BEC (i.e. forming pairs hardly distinguishable from diatomic molecules - Shafroth pairs). Current experiments aim at taking the fermion superfluid into the Bardeen-CooperSchrieffer (BCS) limit of ordinary superconductors, in which the fermion pairs are larger than the average interparticle distance (Cooper pairs). The crossover region [3] , near the detuning of diverging scattering length, is of fundamental interest [4]. The cold atom crossover experiments [5] illustrate the need for unambiguous probes of fermion superfluidity. Sympathetic cooling immerses the fermionic atoms in colder bosonic atoms, often occupying a BEC. The system then resembles the condensed ${ }^{3} \mathrm{He}-{ }^{4} \mathrm{He}$ liquid mixtures [6]. In contrast to the helium liquid mixtures in which strong interaction effects drastically reduce the boson-mediated fermion-fermion interactions [7], the cold atom mixture can exhibit BECmediated fermion superfluidity at attainable temperatures [8].

In this paper, we show that the BEC simultaneously trapped with the fermion atoms, can provide a powerful probe of fermion superfluidity. We study the damping rate, $\Gamma(q)$, of acoustic BEC-excitations (phonons) in the mixture as a function of the phonon momentum $q$. Acoustic attenuation [9] provides a signal for fermion superfluidity, determines the superfluid gap parameter, tests the validity of the weakly interacting quasi-particle description [10], estimates the size of the Cooper-pairs and probes the quasi-particle dispersion relation. The latter could be inferred from kinks which we predict to appear as an imprint of kinematical constraints.

In linear response theory $\Gamma(q)$ is related to densitydensity correlations. For a sufficiently large trap, the damping rate measured near the middle of the trap, where the particle densities are approximately constant, equals that of a BEC-phonon in a homogeneous mixture. The BEC-phonon is a Bogoliubov excitation with en- ergy $E_{q}^{B}=\hbar q c \sqrt{1+(\xi q)^{2}}$, where $c$ denotes the BEC sound velocity, $c=\hbar /\left(2 m_{B} \xi\right), \xi$ is the BEC-healing length, $\xi=\left(16 \pi \rho_{B} a_{B}\right)^{-1 / 2}$, and $a_{B}$ is the boson-boson scattering length. Using box normalization, the boson density $\rho_{B}$ corresponds to $N_{B}$ bosons condensed in a volume $\Omega, \rho_{B}=N_{B} / \Omega$. The system also includes a homogeneous mixture of spin + and - fermions, with $\rho_{+}=\rho_{-}=\rho_{F}$. Each single-component fermion system has Fermi wavenumber $k_{F}=\left(6 \pi^{2} \rho_{F}\right)^{1 / 3}$, Fermi-velocity $v_{F}=\hbar k_{F} / m_{F}$ and Fermi energy $\epsilon_{F}=\hbar^{2} k_{F}^{2} /\left(2 m_{F}\right)$. The boson-fermion interaction is spin dependent, giving different boson-fermion scattering lengths, $a_{+}$and $a_{-}$, and a fermion-boson interaction Hamiltonian $\hat{H}_{\text {int }}=$ $\left(2 \pi \hbar^{2} \bar{a}\right)\left(1 / m_{B}+1 / m_{F}\right) \Omega^{-1} \sum_{\mathbf{k}, j} \hat{\rho}_{-\mathbf{k}}^{B} \alpha_{j} \hat{\rho}_{j, \mathbf{k}}^{F}$, where $j=$ ,$\pm \hat{\rho}_{\mathbf{k}}^{B(F)}$ represents the boson (fermion) density operator, $\bar{a}=\sqrt{a_{-}^{2}+a_{+}^{2}}$, and $\alpha_{j}=\bar{a} / a_{j}$. We use perturbation theory around noninteracting fermion-boson product states, |\rangle$_{F} \otimes|\rangle_{B}$. The $\hat{H}_{\text {int }}$-interaction then annihilates a BEC-phonon $|\mathbf{q}\rangle_{B}$ and creates a fermion excitation that is a collective mode or a quasi-particle pair of opposite spins. By ignoring initial states with multiple BEC excitations, we assume sufficiently low temperatures. By ignoring final states with two (or more) phonons, we make a relative error $\propto(q \xi)^{3}\left(a_{B} / \xi\right)(8 / 3 \pi)$, but we take $(q \xi)<\left(\xi / a_{B}\right)^{1 / 3}$. Using ${ }_{B}\left\langle 0\left|\hat{\rho}_{-\mathbf{q}}\right| \mathbf{q}\right\rangle_{B}=$ $\sqrt{N_{B}\left(\hbar^{2} q^{2} / 2 m_{B}\right) /\left(\hbar \omega_{q}^{B}\right)}, \Gamma(q)$ takes the form $\Gamma(\mathbf{q})=$ $\sum_{i, j} \alpha_{i} \alpha_{j} \Gamma_{i j}(\mathbf{q})$ with

$$
\begin{aligned}
\Gamma_{i j}(\mathbf{q})= & \frac{8}{3} \frac{\left(\Gamma_{0} / 4 \pi\right) \xi q}{\sqrt{1+(\xi q)^{2}}} \int \frac{d^{3} r}{\rho_{F}} \int \frac{d t}{\hbar / \epsilon_{F}} \\
& \times \exp \left[-i\left(\mathbf{q} \cdot \mathbf{r}-\omega_{q}^{B} t\right)\right]\left\langle\hat{\rho}_{i}^{F}(\mathbf{r}, t) \hat{\rho}_{j}^{F}(\mathbf{0}, 0)\right\rangle
\end{aligned}
$$

where $\Gamma_{0}=4 \pi \bar{a}^{2} \rho_{B} v_{F}\left[\left(1+m_{F} / m_{B}\right) / 2\right]^{2}$, proportional to the rate at which a particle of velocity $v_{F}$ scatters off hard-core targets of radius $\bar{a}$, distributed at the BEC density. By varying $a_{+}$or $a_{-}$, one can, in principle, distinguish the $\Gamma_{++}$and $\Gamma_{+-}$contributions. In a Cooper-paired fluid, the $\left\langle\hat{\rho}_{+}^{F} \hat{\rho}_{-}^{F}\right\rangle$ correlations depend on the microscopic superfluid structure, embodied, 
for instance, by the order parameter (i.e. the expectation value of annihilation field pairs, $\left.\left\langle\hat{\psi}_{+} \hat{\psi}_{-}\right\rangle \neq 0\right)$. The equal time \pm -density fluctuation, $\left\langle\hat{\rho}_{+}^{F}(\mathbf{r}) \hat{\rho}_{-}^{F}(\mathbf{0})\right\rangle \approx$ $\left\langle\hat{\psi}_{+}(\mathbf{r}) \hat{\psi}(\mathbf{0})\right\rangle\left\langle\hat{\psi}_{-}^{\dagger}(\mathbf{r}) \hat{\psi}^{\dagger}(\mathbf{0})\right\rangle=F(\mathbf{r}) F^{\star}(-\mathbf{r})$, where $F(\mathbf{r})=$ $\left\langle\hat{\psi}_{+}(\mathbf{r}) \hat{\psi}_{-}(\mathbf{0})\right\rangle$ plays the role of fermion pair wavefunction. Equation (11) also suggests that one can probe directional information if and when cold atom experiments realize anisotropic fermion superfluidity (such as p-wave, d-wave or LOFF-pairing [11, 12]). To make the quasiparticle pair explicit, we insert a summation over such states in the density-density correlation function. The temporal integral gives a delta function that expresses the kinematics of the pair creation process,

$$
\begin{aligned}
& \Gamma_{i j}(\mathbf{q}) \\
& =\frac{\left(8 \Gamma_{0} / 3\right) \xi q}{\sqrt{1+(\xi q)^{2}}} \frac{\epsilon_{F}}{\rho_{F}} \int \frac{d^{3} k}{(2 \pi)^{3}} g_{i, j}(\mathbf{k} ; \mathbf{q}) \delta\left(E_{q}^{B}-E_{k}^{F}-E_{|\mathbf{k}-\mathbf{q}|}^{F}\right)
\end{aligned}
$$

where $E_{k}^{F}$ denotes the quasi-particle dispersion relation and $g_{i, j}(\mathbf{k} ; \mathbf{q})$ is the weight for the creation of quasiparticles of momenta $\mathbf{k}$ and $\mathbf{q}-\mathbf{k}$. The generalized Wick's theorem leads to $g_{++}=g_{--}=n_{\mathbf{k}}\left(1-n_{\mathbf{q}-\mathbf{k}}\right)$, and $g_{+-}=$ $g_{-+}=F_{\mathbf{k}} F_{\mathbf{q}-\mathbf{k}}^{*}$, with $F(\mathbf{r})=(2 \pi)^{-3} \int d^{3} k F_{\mathbf{k}} \exp (i \mathbf{k} \cdot \mathbf{r})$, and $n_{\mathbf{k}}=\left\langle c_{j, \mathbf{k}}^{\dagger} c_{j, \mathbf{k}}\right\rangle$. Here, $c\left(c^{\dagger}\right)$ denote fermion annihilation (creation) operators.

The form of Eq. (2) is quite general but the lack of correlations resulting from Wick's rule, is not. We can understand the role of $F$ as pair wavefunction by comparing Eq. (2) to the Fermi Golden-rule rate at which a particle of momentum $\mathbf{q}$ and energy $E_{q}^{B}$ scatters to zero momentum by breaking the bond of stationary diatomic molecules of $(+)$ and $(-)$ atoms. In the initial molecules, these atoms occupy bound vibrational states with wavefunction $\varphi_{\mathbf{k}}$ and binding energy $E_{b}$. The interaction that breaks the molecular bond, $\hat{H}_{\text {int }}$, creates a pair of free atoms, the $(+)$ atom with momentum $\mathbf{k}$ and energy $e_{k}$, and the $(-)$ atom with momentum $\mathbf{q}-\mathbf{k}$ and energy $e_{|\mathbf{q}-\mathbf{k}|}$. The corresponding interaction matrix element is proportional to $\alpha_{-} \varphi_{\mathbf{k}}+\alpha_{+} \varphi_{\mathbf{k}-\mathbf{q}}$, so that the sizedependent contribution to the rate is

$$
\frac{1}{\tau_{q}} \propto \alpha_{+} \alpha_{-} \int d^{3} k \varphi_{\mathbf{k}} \varphi_{\mathbf{k}-\mathbf{q}}^{*} \delta\left(E_{q}-E_{b}-e_{k}-e_{|\mathbf{k}-\mathbf{q}|}\right),
$$

which has the form of Eq. (2) if we associate $F_{\mathbf{k}} \rightarrow \varphi_{\mathbf{k}}$ and $E_{\mathbf{k}}^{F} \rightarrow E_{b} / 2+e_{k}$. Similar to the $g_{+-}$contribution to Eq. (2), Eq. (3) stems from an interference of two processes in reaching the final state. In the first process, atom $(+)$ with momentum $\mathbf{k}$ and atom $(-)$ with momentum $-\mathbf{k}$ in the molecule reach the final state because the $(-)$ atom receives a momentum kick $\mathbf{q}$. In the second process, the $(+)$ and $(-)$ atoms in the molecule have initially momenta $\mathbf{k}-\mathbf{q}$ and $\mathbf{q}-\mathbf{k}$, respectively, and the $(+)$ atom receives a momentum kick $\mathbf{q}$.

The role of the fermion density of states, $D(E)=$ $\left(2 \pi^{2}\right)^{-1} k^{2}(E) /\left|\partial E^{F} / \partial k\right|$, becomes apparent when we convert Eq. (2) to an integral over quasi-particle energy. Targeting s-wave pairing, we assume a spherically symmetric pairing wavefunction, $F_{\mathbf{q}}=F_{q}$. Introducing the angle $\theta$ between $\mathbf{k}$ and $\mathbf{q}$, we write

$$
\begin{array}{r}
\delta\left[E_{q}^{B}-E^{F}(k)-E^{F}\left(\sqrt{k^{2}+q^{2}-2 k q \cos \theta}\right)\right] \\
=\sum_{j} \frac{|\mathbf{q}-\mathbf{k}|_{j} \delta\left(\cos \theta-\cos \theta_{j}\right)}{k_{j} q|\partial E(\kappa) / \partial \kappa|_{\kappa=|\mathbf{q}-\mathbf{k}|_{j}}},
\end{array}
$$

where the $j$-subscript enumerates the energy and momentum conserving quasi-particle pairs. Whether or not the fermion dispersion relation supports a local minimum (i.e., whether or not the fermion superfluid resides in the BCS regime), affects the number of such pairs. If $E_{k}^{F}$ has a local minimum, we refer to it as the "gap" $\Delta$, $E_{k_{m}}^{F}=\Delta$ and we refer to the corresponding surface in momentum space, $k=k_{m}$, as the gap surface. We can then assign two momentum magnitudes for the same quasi-particle energy $E$ : $k_{<}(E)$ inside the gap surface $\left(k_{<}(E)<k_{m}\right)$ and $k_{>}(E)$ outside $\left(k_{>}(E)>k_{m}\right)$. The phonon annihilation can generate four quasi-particle pair types, $j=(<,<),(<,>),(>,<),(>,>)$, where we indicate the momentum position of the $(+)$ particle first, and that of the (-) particle second. Depending on the energy distribution, some pair creation processes $j$ are excluded kinematically. We can picture the constraints geometrically for the j-pair creation by considering the spherical surfaces in momentum space of radii $k_{j,+}(E)$ and $k_{j,-}\left(E^{\prime}\right)$, with $E+E^{\prime}=E_{q}^{B}$. As the $(+)$ momentum $\mathbf{k}$ lies on the first surface, the (-) momentum $\mathbf{q}-\mathbf{k}$ on the second, the surfaces must be connected by the q-momentum, requiring $k_{j,+}(E)+k_{j,-}\left(E^{\prime}\right)<q<\left|k_{j,+}(E)-k_{j,-}\left(E^{\prime}\right)\right|$. Then, the damping rates read

$$
\begin{aligned}
& \frac{\gamma+-(q)}{\left(\frac{8 \pi^{2} \xi \epsilon_{F}}{3 \rho_{F}}\right)}=\sum_{j} \int d E \frac{D_{j}(E) F_{j}(E)}{k_{j}(E)} \frac{D_{j}\left(E^{\prime}\right) F_{j}\left(E^{\prime}\right)}{k_{j}\left(E^{\prime}\right)} \\
& \frac{\gamma_{++}(q)}{\left(\frac{8 \pi^{2} \xi \epsilon_{F}}{3 \rho_{F}}\right)}=\sum_{j} \int d E \frac{D_{j}(E) n_{j}(E)}{k_{j}(E)} \frac{D_{j}\left(E^{\prime}\right)\left[1-n_{j}\left(E^{\prime}\right)\right]}{k_{j}\left(E^{\prime}\right)}
\end{aligned}
$$

where $\gamma_{i j}(q)=\left[\Gamma_{i j}(q) \sqrt{1+(\xi q)^{2}}\right] / \Gamma_{0}$. What is the information content of $\Gamma(q)$ ? The gap prevents soft phonons with $E_{q}^{B}<2 \Delta$ from damping through pair excitation. If these soft phonons are kinematically prohibited from exciting collective fermion modes because the latter's velocity ( $\sim v_{F}$ for the AndersonGoldstone mode in the BCS limit) exceeds $c$, then they can only decay through Beliaev damping which gives a negligible damping rate at long wavelengths $\left(\Gamma_{\text {Beliaev }}(q) \sim(6 \sqrt{\pi} / 5) \sqrt{\rho_{B} a_{B}^{3}}(q c)(q \xi)^{4}\right)$. More energetic BEC phonons damp by radiating quasi-particle pairs, and the higher the density of states, the more an energy interval contributes to the damping. At the gap surface the density of states is infinite, although the divergence is sufficiently slow $\left(\propto \sqrt{E^{2}-\Delta^{2}}\right)$ to ensure that 


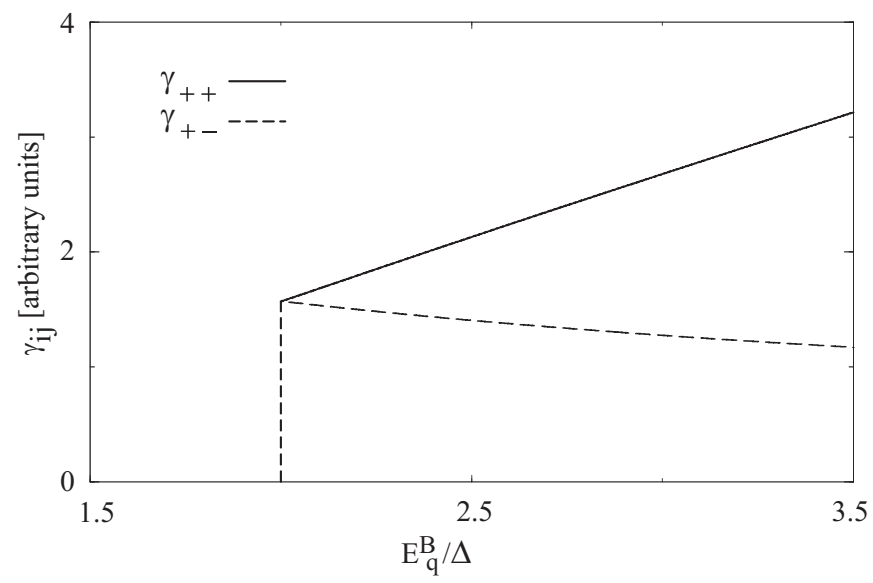

FIG. 1: Scaled damping rates components, $\gamma_{++}$and $\gamma_{--}$, in the BCS limit, as a function of the phonon energy, $E_{q}^{B}$, measured in units of $\Delta$, the superfluid gap parameter. Here, we have $\gamma_{i j}=\Gamma_{i j} \sqrt{1+(\xi q)^{2}} / \Gamma_{0}$.

the integral (5) remains finite. The divergence plays a prominent role when both quasi-particles form on or near the gap surface, corresponding to a phonon energy that slightly exceeds $2 \Delta$. While the damping rate remains finite, the diverging density of states causes a discontinuous jump of the damping rate at the (edge) phonon momentum $q_{\Delta}$ for which $E_{q_{\Delta}}^{B}=2 \Delta$, see Fig. [1 Conversely, the discontinuity in the damping rate points towards a diverging density of states, providing a signature of fermion superfluidity on the BCS side of the crossover. This sharp feature also provides a sensitive way of determining the superfluid gap from the edge momentum $q_{\Delta}$, $\Delta=\left(\lambda_{B} \rho_{B}\right)\left(\xi q_{\Delta}\right) \sqrt{1+\left(q_{\Delta} \xi\right)^{2}}$. On the other hand, the observation of a continuous onset of the damping with increasing $q$ does not necessarily imply that the fermions, if superfluid, have entered the BEC-side of the crossover. Indeed, as the system nears the crossover from the BCS side in an adiabatic sweep through a Feshbach resonance, we expect $k_{m}$ to continuously tend to zero, whereas $q_{\Delta}$ monotonically increases. Beyond the detuning for which $k_{m}=q_{\Delta}, q_{\Delta}$ is too large to fit inside the gap surface. In that case, the creation of both quasi-particles on the gap surface, the event that leads to the discontinuous jump in damping rate, is kinematically prohibited. The dependence of the damping rate on Cooper-pair size, suggested by the analogy with breaking the stationary molecule bond, Eq. (3), becomes apparent when we expand the damping rates near the edge. We make two assumptions: (i) $\left(E_{k}^{F}\right)^{2} \approx \Delta^{2}+\hbar^{2} v^{2}\left(k-k_{m}\right)^{2}$, in which $v$ characterizes the curvature of the dispersion near the gap. At $k=k_{m}$, $F\left(k_{m}\right)=F_{m}$ and $n\left(k_{m}\right)=n_{m}$. (ii) The fermion system is sufficiently far from the crossover point to ensure that all four quasi-particle pair events are kinematically allowed.
Therefore, the damping rates near $q=q_{\Delta},\left(q>q_{\Delta}\right)$ are

$$
\begin{aligned}
& \frac{\gamma_{+-}(q)}{2 \pi^{2}\left(\xi k_{F}\right)\left(\Delta /\left[m_{F} v^{2} / 2\right]\right)} \frac{1}{F_{m}^{2}} \\
& \approx 1+\frac{E_{q}^{B}-2 \Delta}{\Delta}\left[\frac{3}{4}+\left.\frac{\Delta^{2}}{(\hbar v)^{2}} \frac{\nabla_{k}^{2} F_{k}}{F_{k}}\right|_{k=k_{m}}\right] \\
& \frac{\gamma_{++}(q)}{2 \pi^{2}\left(\xi k_{F}\right)\left(\Delta /\left[m_{F} v^{2} / 2\right]\right)} \frac{1}{n_{m}\left(1-n_{m}\right)} \\
& \approx 1+\frac{E_{q}^{B}-2 \Delta}{\Delta}\left[\frac{3}{4}+\left.\frac{\Delta^{2}}{(\hbar v)^{2}} \frac{1-2 n_{m}}{1-n_{m}} \frac{\nabla_{k}^{2} n_{k}}{n_{k}}\right|_{k=k_{m}}\right] .
\end{aligned}
$$

Note that the ratio of the edge values of the $(+,+)$ and $(+,-)$ damping rates connect $n_{m}$ and $F_{m}$ : $\Gamma_{+-}\left(q_{\Delta}\right) / \Gamma_{++}\left(q_{\Delta}\right)=n_{m}\left(1-n_{m}\right) / F_{m}^{2}$. This ratio equals one in a mean-field description for short-range pairing interactions because then $F_{m}=n_{m}=1 / 2$. This ratio can deviate significantly from one when long-range interactions are present, particularly in the crossover region. If $F_{m}=n_{m}=1 / 2$ and the $(+,+)$ and $(+,-)$ edge damping rates are identical, then one can measure $v$ from their absolute value, since these are proportional to $\left(m v^{2} / 2 \Delta\right)$ and $\Delta$ follows from $q_{\Delta}$. From the slope of the scaled damping rate $\gamma_{+,-}(q)$, set out as a function of the phonon energy $E_{q}^{B}$, one can extract the square of the coherence length, $-\nabla_{k}^{2} F_{k} /\left.F_{k}\right|_{k=k_{m}}=$ $\int d^{3} r F(r) r^{2} \exp \left(i \mathbf{k}_{m} \cdot \mathbf{r}\right) / \int d^{3} r F(r) \exp \left(i \mathbf{k}_{m} \cdot \mathbf{r}\right)$, where $\left|\mathbf{k}_{m}\right|=k_{m}$. Near crossover, where the extent of $F$ drops below $k_{m}^{-1}$, this coherence length tends to the range of $F$. In the BCS-limit, $k_{m} \rightarrow k_{F}, v \rightarrow v_{F}$ and $-\nabla_{k}^{2} F_{k} /\left.F_{k}\right|_{k=k_{m}} \rightarrow\left(\hbar v_{F} / \Delta\right)^{2}$ so that the slope tends to $-1 / 4$, as shown in Fig. 1] Near the crossover $q_{\Delta}$ grows and, therefore, it is likely that the kinematical condi-

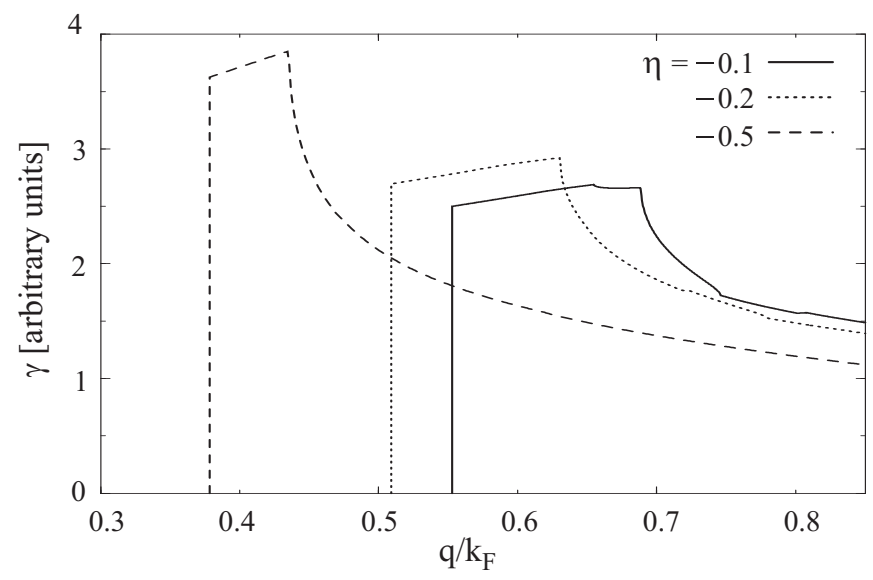

FIG. 2: Near-edge pair breaking contribution to the scaled damping rate $\gamma\left(\gamma=\Gamma \sqrt{1+(\xi q)^{2}} / \Gamma_{0}\right)$, as a function of the phonon momentum, $q$. Here we assumed $a_{+}=a_{-}, c / v_{F}=$ 0.5 and varied the fermion-fermion scattering lengths $a_{F}$ or $\eta=\left(k_{F} a_{F}\right)^{-1}$. The edge momentum gives the superfluid gap, the slope near the edge relates to the size of the Cooper-pairs and the discontinuities in the derivatives (kinks) are imprints of the kinematical constraints of the pair breaking process. 
tions $\left|k_{j,+}(E)-k_{j,-}\left(E^{\prime}\right)\right|<q<k_{j,+}(E)+k_{j,-}\left(E^{\prime}\right)$ are violated near the edge. At the minimal phonon energy $E_{q}^{B}$ for which quasi-particle pairs $j$ are kinematically excluded, the damping curve exhibits a kink. We expect the kinks to be smoothed out if the quasi-particles have finite lifetimes. In Fig. 2, we show $\Gamma(q)$ for a zero temperature Cooper-paired system at different detuning values during an adiabatic sweep through the resonance. The curves were calculated from a Keyldish-like wavefunction, $e^{-\alpha \sum_{k} \phi_{k} c_{\mathbf{k},+}^{\dagger} c_{-\mathbf{k},-}^{\dagger}}|0\rangle$, where we choose $\phi_{k}$ as a Yukawa wavefunction with the range given by the BCS coherence length, $R=\Delta / \hbar v_{F}$. While the assumed wavefunction cannot describe the system quantitatively, the resulting damping rates illustrate the relevant physics.

To demonstrate the practicality of cold atom acoustic attenuation, we consider a realistic two-component fermion $\left({ }^{6} \mathrm{Li}\right)-\mathrm{BEC}\left({ }^{23} \mathrm{Na}\right)$ mixture $\left(a_{B}=3.4 \mathrm{~nm}, \xi=0.14 \mu \mathrm{m}\right)$, at temperature $T=50 \mathrm{nK}$, trapped by harmonic oscillator potentials within a radius $R_{0}=50 \mu \mathrm{m}$ with peak densities $\rho_{F}=10^{12} \mathrm{~cm}^{-3}$ and $\rho_{B}=3 \times 10^{14} \mathrm{~cm}^{-3}$. We assume that $\bar{a}=5 \mathrm{~nm}$ and that the system has a gap $\Delta \sim \epsilon_{F} / 5$. For these parameters, $\lambda_{B} \rho_{B}=266 \mathrm{nK}$, $\epsilon_{F}=610 \mathrm{nK}(\Delta=122 \mathrm{nK})$, the relevant velocities are $v_{F}=4 \mathrm{~cm} / \mathrm{sec}$ and $c=1 \mathrm{~cm} / \mathrm{sec}$, the time scale for BECphonon scattering is $\Gamma_{0}^{-1}=0.17 \mathrm{msec}$ during which time the BEC-excitation travels a distance $\Gamma_{0}^{-1} c \sim 1.7 \mu \mathrm{m}$, and the critical phonon momentum for pair breaking, $q_{\Delta}$, (obtained from $\left(q_{\Delta} \xi\right)^{2}=-1 / 2+\sqrt{1 / 4+\left[\Delta /\left(\lambda_{B} \rho_{B}\right)\right]^{2}} \sim$ 0.178 ) is equal to $q_{\Delta}=3 \mu \mathrm{m}^{-1}$. Creating the wavepacket by overlapping two laserbeams over a region $L$ of $5 \mu \mathrm{m}$ gives a relative momentum spread $\delta q / q_{\Delta} \sim 1 /\left(L q_{\Delta}\right) \sim$ 0.067 . Measuring the phonon damping or scattering after a time less than or equal to $0.5 \mathrm{msec}$, this wavepacket would have traveled over less than $5 \mu \mathrm{m}$, so that the measurement can be carried out within a radius $L_{\text {meas }}=$ $5 \mu \mathrm{m}$ [13]. The relative density variations, only one percent for $L_{\text {meas }} / R_{0} \sim 0.1$ since $\delta \rho_{B} / \rho_{B} \sim\left(L_{\text {meas }} / R_{0}\right)^{2}$, $\delta \rho_{F} / \rho_{F} \sim(3 / 2)\left(L_{\text {meas }} / R_{0}\right)^{2}$, smooth out the sharp features of the damping curve but only over a few precent of the phonon momentum, less than the relative momentum uncertainty due to the finite size of the wavepacket $\sim 5-7 \%$. Even with a smoothing of ten percent of the momentum, we expect the most abrupt kinks shown in Fig. 2 to be clearly visible. At finite temperature, thermal scattering contributes a smooth background that is considerably smaller than the pair breaking term at sufficiently low temperatures, such as in the above example. At $\Delta<k_{B} T$, the thermal occupation of fermion quasiparticles is exponentially suppressed and so is the corresponding contribution to the phonon scattering. The scattering by thermally excited BEC phonons is not suppressed, but still small: estimating its magnitude roughly as $\gamma_{\text {therm }} \sim 4 \pi \bar{a}^{2} \rho_{B} c_{B} f$ where $f$ is the fraction of thermally depleted bosons, $f \sim 0.02$ (from $f \sim\left(T / T_{\mathrm{BEC}}\right)^{3 / 2}$, where the $\mathrm{BEC}$ critical temperature, $T_{\mathrm{BEC}} \sim 20 \mu \mathrm{K}$ at $\rho_{B}=3 \times 10^{14} \mathrm{~cm}^{-3}$ ) gives $\gamma_{\text {therm }} \sim 0.03 \Gamma_{0}$.

In summary, we have shown that the damping rate of an acoustic excitation of an overlapping BEC can probe the superfluidity of an ultra-cold fermionic gas. The momentum dependence of this rate yields an unambiguous signature of BCS-like superfluidity, allows a measurement of the superfluid gap parameter and of the correlation length in the BEC-BCS crossover regime. Kinks in the momentum dependence of the damping rate will reveal information about the quasi-particle dispersion relations.

* Now at Universita' degli Studi di Roma, La Sapienza, and Institute of Complex Systems, CNR, Roma, 00185, Italy

Electronic address: gaudios@roma1.infn.it

† Electronic address: eddy@lanl.gov

[1] M. Greiner, C.A. Regal, D.S. Jin, Nature (London) 426, 537 (2004); M. W. Zwierlein et al., Phys. Rev. Lett. 91, 250401 (2003).

[2] Except for certain ceramic systems, see R.J. Tainsh, C. Andrikidis, Solid State Commun. 60, 517 (1986) ; D.M. Eagles, Solid State Commun. 60, 521 (1986).

[3] D.M. Eagles, Phys. Rev. 186, 456 (1969); A.J. Leggett, in Modern Trends in the Theory of Condensed Matter, edited by A. Pekalski and R. Przystawa, Springer Verlag, Berlin, 1980; C.A.R. Sa de Melo, M. Randeria, and J.R. Engelbrecht, Phys. Rev. Lett. 71, 3202 (1993).

[4] Superfluid properties play a crucial role in neutron star cooling, e.g. D.G. Yakovlev, and C.J. Pethick, Annu. Rev. Astrophys. 42, 169 (2004).

[5] C. Chin et al., Science 305, 1128 (2004); J. Kinast et al. Phys. Rev. Lett. 92150402 (2004); J. Kinast, A. Turlapov, and J.E. Thomas, Phys. Rev. Lett. 94, 170404 (2005); G.B. Partridge et al., Phys. Rev. Lett. 95, 020404 (2005); J. Kinast et al., Science 37 (2005); M. Bartenstein et al., cond-mat/0412712.

[6] E. Krotscheck, and M. Saarela, Phys. Rep. 232, 1 (1993).

[7] J. Bardeen, G. Baym, and D. Pines, Phys. Rev. 256, 207 (1967).

[8] H. Heiselberg et al., Phys. Rev. Lett. 85 (12), 2418 (2000).

[9] J.R. Schrieffer, Theory of Superconductivity (Frontiers in Physics, Reading, MA, 1964).

[10] Fluctuations can be prominent, see S. Gaudio, J. Jackiewicz, and K.S. Bedell, cond-mat/0505306

[11] P. Fulde and R.A. Ferrell, Phys. Rev. 135 A550 (1964); A.I. Larkin and Yu.N. Ovchinnikov, Zh. Eksp. Teor. Fiz. 47, 1136 (1964) [Sov. Phys. JETP 20, 762 (1965)].

[12] M.M. Forbes et al., Phys. Rev. Lett, 94, 017001 (2004).

[13] Trap experiments measured bulk-like quantities by observing a localized excitation over a time less than the transit time: A. P. Chikkatur et al., Phys. Rev. Lett. 85, 483 (2000); J.M. Vogels et al., Phys. Rev. Lett. 88, 060402 (2002). 